Supporting Information

\title{
Intramolecular and Intermolecular Packing in Polymer Crystallization
}

Shijun Wang, ${ }^{1}$ Shichen Yuan, ${ }^{1}$ Kun Wang, ${ }^{1}$ Wei Chen, ${ }^{2}$ Koji Yamada, ${ }^{3}$ Deborah Barkley, ${ }^{4}$ Tadanori Koga, ${ }^{4,5}$ You-lee Hong, ${ }^{6}$ and Toshikazu Miyoshi ${ }^{1, *}$

${ }^{1}$ Department of Polymer Science, The University of Akron, Akron, Ohio 44325-3909, USA. ${ }^{2}$ National Synchrotron Radiation Lab, CAS Key Laboratory of Soft Matter Chemistry, Anhui Provincial Engineering Laboratory of Advanced Functional Polymer Film, University of Science and Technology of China, Hefei, China.

${ }^{3}$ Advanced Processing Technology Unit Research Center, Toyobo Co., Ltd. 2-1-1 Katata, Otsu, Shiga 520-0292, Japan. ${ }^{4}$ Department of Chemistry and ${ }^{5}$ Department of Materials Science and Chemical Engineering, Stony Brook University, Stony Brook, New York 11794.

${ }^{6}$ RIKEN CLST-JEOL Collaboration Center, RIKEN, Yokohama, Kanagawa 230-0045, Japan. 

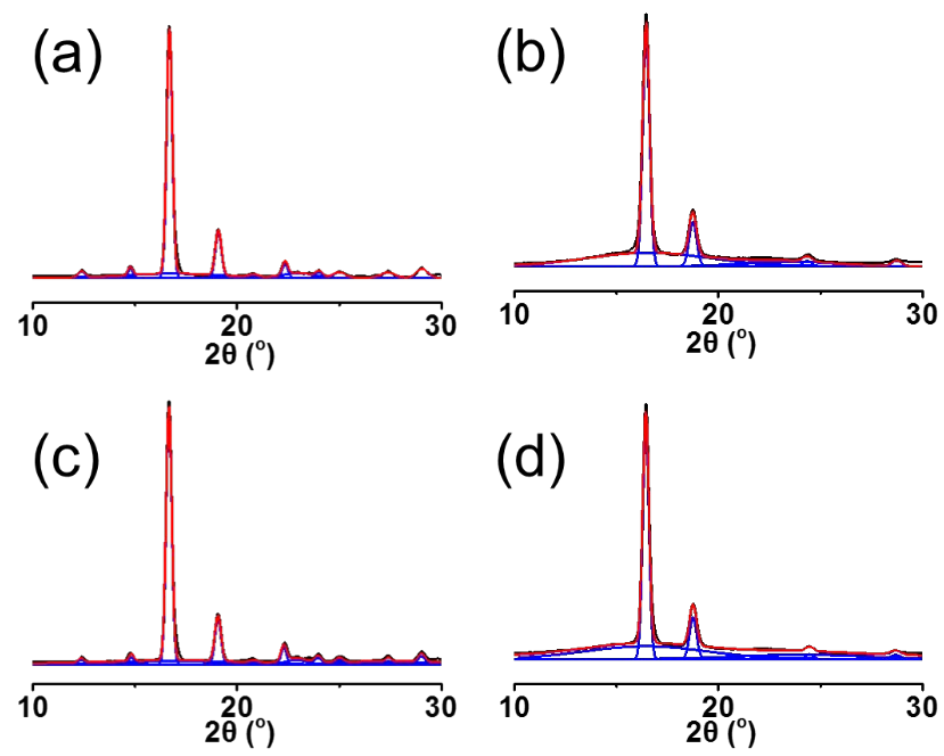

Figure S1. The WAXD profiles (black lines) and the best-fit Gaussian peaks (blue and red lines) for $s$ - (a and b) and $l$-PLLA (c and d) melt-grown crystals at $T_{\mathrm{c}}=150{ }^{\circ} \mathrm{C}\left(\mathrm{a}\right.$ and $\mathrm{c}$ ) and $90{ }^{\circ} \mathrm{C}(\mathrm{b}$ and d).

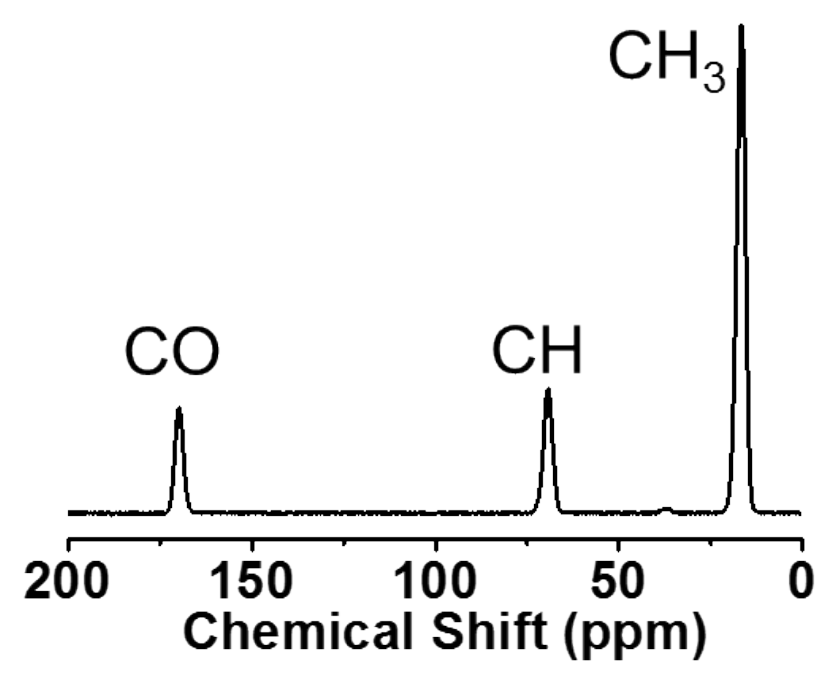

Figure S2. ${ }^{13} \mathrm{C}$ CPMAS NMR spectrum for $m$-PLLA labeled and non labeled samples with 1:9 blending ratio measured at a MAS frequency of $10 \mathrm{kHz}$. 

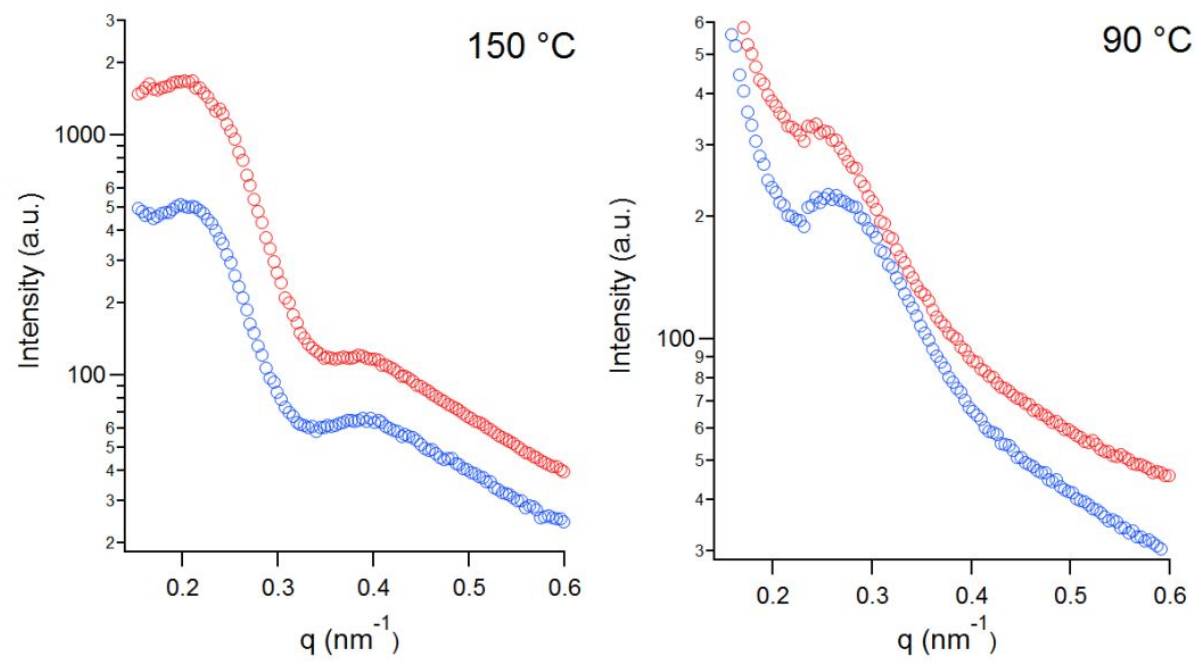

Figure S3. SAXS patterns for $s$ - (blue open circle) and $l$-PLLA (red) at $T_{\mathrm{c}}=90$ and $150{ }^{\circ} \mathrm{C}$.

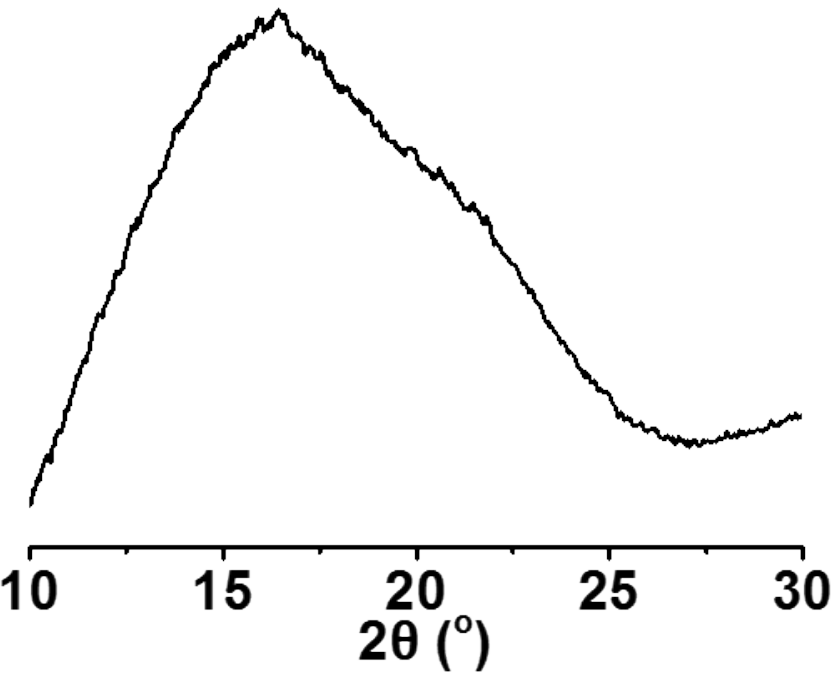

Figure S4 WAXD profile for the $m$-PLLA glasses. 